\title{
Potential of Sustained Release Microparticles of Metformin in Veterinary Medicine: An in Vivo Pharmacokinetic Study of Metformin Microparticles as Oral Sustained Release Formulation in Rabbits.
}

\section{Sihem Bouriche}

Universite de Bejaia Faculte de Technologie

Angela Alonso-García

Universidad de Murcia Faculta de Veterinaria

Carlos M Cárceles-Rodríguez ( $\nabla$ carceles@um.es )

University of Murcia: Universidad de Murcia https://orcid.org/0000-0002-9819-280X

\section{Farouk Rezgui}

Universite de Bejaia Faculte de Technologie

Emilio Fernández-Varón

Universidad de Murcia Faculta de Veterinaria

\section{Research article}

Keywords: Controlled-release, microparticles, pharmacokinetics, poly (lactic acid), In Vitro/In Vivo (IVIVC) Correlation(s)

Posted Date: October 12th, 2020

DOI: https://doi.org/10.21203/rs.3.rs-80177/v1

License: (c) (i) This work is licensed under a Creative Commons Attribution 4.0 International License.

Read Full License 


\section{Abstract}

Background: Metformin hydrochloride a biguanide derivative has been widely used in the treatment of type 2 diabetes in humans. In veterinary medicine, metformin has been increasing his potential in different species as equids for insulin dysregulation, dogs and cats with diabetes. It is a highly soluble hydrophilic drug, shows incomplete absorption from the gastrointestinal tract and the absolute bioavailability is $40-60 \%$ with a short biological half-life of 1.5-1.6 h in humans. In this study, to improve its efficacy a sustained-release microparticles of metformin was developed by loading within poly lactic acid (PLA) polymer followed by an in vivo pharmacokinetics study in rabbits.

Results: Pharmacokinetic study in rabbits showed the sustained-release characteristic from the prepared microparticles with delayed time to reach maximum concentrations $\mathrm{T}_{\text {max }}$, decreased $\mathrm{C}_{\max }$, increased Mean Residence Time (MRT) and half-life compared to the pure drug solution. Physicochemical characterization suggested that PLA and metformin hydrochloride interacted within the microparticles via hydrogen bonds and that the drug was transformed to an amorphous state.

Conclusions: The The pharmacokinetics parameters resulted in delayed $T_{\text {max }}$, increased MRT and $t_{1 / 2}$, decreased $\mathrm{C}_{\max }$ of metformin from microparticles that show promise for prolonged/sustained release of metformin after oral administration in different animal species affected by insulin disorders. PLA microparticles provided sustained release of the drug, and these systems can be useful as drug carriers for hydrophilic drugs in long term disease treatment such as diabetes.

\section{Background}

Diabetes mellitus is a chronic problem in humans characterised by high blood glucose levels induced by; a deficient secretion of insulin, anomalous of insulin action or both. It is a main factor of developing health complications such as cardiovascular disease, neuropathy, nephropathy and eye disease, leading to retinopathy and blindness. Diabetes is classified into three main types; gestational diabetes, type 1diabetes and type 2-diabetes. The latest one represents $90 \%$ of all the cases [1].

In veterinary medicine, diabetes affects to different animal species, it is more frecuently reported in dogs and cats [2], and rarely in other animal species like rabbits [3]. In equids, insulin dysregulation is the primary endocrine disorder in equine metabolic syndrome (EMS) affecting also some equids with pituitary pars intermedia dysfunction (10-22\% prevalence) $[4,5]$. There are several reports about the use of the hypoglycemic drug metformin in clinical cases in different animal species [2, 4]. However, its efficacy is controversial mainly due to its low oral bioavailability [2, 4].

Metformin hydrochloride (metformin, Fig. 1.A) a biguanide derivative of galegine alkaloid, is used as a first line treatment for type 2 diabetes. In the presence of insulin, it acts by lowering the blood glucose concentration mainly through the inhibition of the gastrointestinal glucose absorption and the hepatic glucose production, increasing the glucose uptake and insulin sensitivity in muscle [6]. Metformin a highly soluble hydrophilic drug with low permeability via biological membrane belongs to 
Biopharmaceutics Classification System class III drug (BCS).The low permeability of this drug results in poor drug absorption which is the rate limiting step in attaining suitable bioavailability. After oral administration, metformin is incompletely absorbed from the gastrointestinal tract. It has $40-60 \%$ absolute bioavailability with a short biological half-life (1.5-1.6 h) [7]. To maintain effective plasma concentration, it should repeatedly be taken at high doses $(2.5 \mathrm{~g} /$ day) with a consequent of serious gastrointestinal side effects [8]. Furthermore, metformin is required to be taken for a long period by patients.

In such therapeutic treatment, the development of polymeric controlled drug delivery systems (CDDSs) is an important strategy to overcome the above limitations. The purpose of these systems is to reduce side effects, sustain drug release and increase the bioavailability of drugs at a controlled rate for a sustained time (days, weeks or months) [9]. Thereby the drugs concentration is maintained between the therapeutic windows over a specified period of time. In this context, microparticles formulated with polymers, are intensively used in designing CDDSs for a various therapeutic drugs $[10,11]$.

Poly lactic acid (PLA, Fig. 1.b) is a hydrophobic polymer belongs to the a-hydroxyl acid family. As a biodegradable and biocompatible, this polymer is widely used for many applications in pharmaceutical and medical areas because its decomposition products are not toxic and are easily excreted from human organism $[12,13]$. Several methods for the preparation of CDDs microparticles from PLA polymer were reported in the literature. ${ }^{8}$ Because of the easy preparation process, solvent evaporation method (double emulsion) is mostly used to encapsulate water soluble drugs.

The literature does not indicate studies involving microparticles containing metformin, using PLA as polymer. Recently, an optimisation of metformin encapsulation/formulation in PLA microparticlesin terms of encapsulation efficiency was investigated by using response surface design [14]. However, in vivo bioavailability of the prepared microparticles was not done. It is of important to investigate the in vivo performance of the formulated microparticles formulation. For this purpose, this study was conducted to investigate the in vivo metformin sustained release and the pharmacokinetics parameters of PLA microparticles after oral administration to rabbits and compared to pure drug solution administered by oral and IV routes.

\section{Results}

\section{Encapsulation efficiency and characterization of PLA microparticles}

Metformin $\mathrm{HCl}$ was encapsulated within PLA microparticles by double emulsion solvent evaporation method. Many batches were prepared; the percentage of encapsulation efficiency of metformin calculated was $76 \pm 2.72 \%$. 
The obtained microparticles were analyzed by scanning electron microscopy (SEM). SEM micrograph of the PLA encapsulated metformin is shown in Fig. 2. The microparticles had almost spherical shapewith smooth surfaces with particles adhered to the surface. In addition to surface morphology characteristic, it is interesting to note that the size of microparticles is variable, it is ranged from $\sim 1$ to $55 \mu \mathrm{m}$. SEM images also suggested that metformin was dissolved in the microparticles, since no individualized drug crystals observed.

\section{Fourier transforms infrared (FTIR) spectroscopy}

FTIR spectroscopy was used to investigate the encapsulation process of metformin loaded microparticles. FTIR spectra of pure metformin, blank PLA MPs and metformin loaded microparticles are shown in Fig. 3. The FTIR spectrum of pure metformin (Fig. 3a) shows characteristic peaks at 3390, 1633 and $1045 \mathrm{~cm}^{-1}$ corresponds to the stretching of primary $\mathrm{N}-\mathrm{H}$, stretching of $\mathrm{C}=\mathrm{N}$, and stretching of $\mathrm{C}-\mathrm{N}$ respectively. For the blank MPs (Fig. 3b) four characteristic peaks were observed at 1764, $2993-2943$ and 1087 which are attributed to $\mathrm{C}=\mathrm{O}$ stretching of the ester group, asymmetric and symmetric vibration of the $\mathrm{CH} 3$ group $-\mathrm{CH}_{3}$ (asym), $\mathrm{CH}_{3}$ (sym) and $\mathrm{C}-0$ ester bond.As shown in the microparticles spectra (Fig. 3c), the intensity of the peak of $\mathrm{C}=0$ ester groups of PLA at $1764 \mathrm{~cm}^{-1}$ after metformin loading was reduced, this is due to hydrogen bond formation between carbonyl of ester of PLA and hydrogen of amine groups of metformin (Fig. 1). It is reported that the elongation of $\mathrm{C}=\mathrm{O}$ band to $\mathrm{C}-\mathrm{O}-\mathrm{H}$ band after hydrogen bond formation resulted in shift of absorption band to lower wave number. These results confirm that metformin was successfully encapsulated in PLA microparticles.

\section{X-ray powder diffraction (XRPD)}

Figure 4 illustrates the X-ray diffraction (PXRD) difractogram of pure metformin, blank MPs and metformin loaded microparticles. The PXRD difractogram of Met revealed the distinct peaks at 20: $12.17 \circ, 17.62 \circ, 24.47 \circ, 28.2 \circ, 31.17 \circ, 37.07 \circ$; these sharps peaks confirmed the highly crystalline nature of metformin. Blank MPs showed the peaks at 20: 16.6०, 18.92。 indicating the semi crystalline nature of the PLA [15]. The PXRD difractogram of MPs shows no peaks. As observed in Figure (4), the peaks of both PLA and metformin are disappeared as compared to Blank MPs and pure metformin. This may be attributed to the incorporation of metformin into PLA matrix leading to a change in the crystallinity of the PLA and metformin. The results showed a good entrapment of Met in PLA with interaction which are aligned with FT-IR analysis.

\section{Differential scanning calorimetry (DSC)}

In addition to IRTF and XRD, DSC analysis was performed to obtain more results about the physical state of metformin in PLA microparticles and intermolecular interactions between the drug and the polymer. The DSC curves of samples were recorded during the first heating process Fig. 5. The metformin DSC curve exhibited a very sharp endothermic peak at $\sim 232{ }^{\circ} \mathrm{C}$ that indicates its crystalline nature. DSC curve of the blank PLA (unloaded) microparticles showed a broad peak at $\sim 178{ }^{\circ} \mathrm{C}$, while this peak is shifted to $168^{\circ} \mathrm{C}$ in metformin loaded PLA microparticles. No peak of metformin in the loaded microparticleswas recorded. The water soluble metformin has interaction with the PLA via the electrostatic force between 
$\mathrm{NH}^{+}$and $\mathrm{COO}^{-}$, consequently changes in the thermal behaviour of both metformin and PLA polymer are occurred [16]. This result indicates that metformin is dispersed or dissolved into the polymer matrix during microencapsulation process.

\section{In vivo Pharmacokinetic studies in rabbits}

The in vivo study was conducted to assess the pharmacokinetics of metformin loaded PLA microparticles after three routes of administration (intravenous solution, oral solution and oral PLA microparticles). The mean plasma concentration-time profiles after a single administration at the same dose $(5 \mathrm{mg} / \mathrm{kg}$ ) to rabbits of the IV, oral solutions and the oral microparticles of metformin are illustrated in Fig. 6. Quantification of metformin in plasma after $24 \mathrm{~h}$ was not detected by HPLC. No adverse events were observed during the study.

After oral administration of pure metformin solution, the drug was absorbed gradually to reach a maximum of absorption at a $T_{\max }$ of $110 \mathrm{~min}$ with a higher $C_{\max }$ of $3.19 \pm 0.16 \mu \mathrm{g} / \mathrm{ml}$ followed by a decrease to $\sim 1 \mu \mathrm{g} / \mathrm{mL}$ within 300 minutes. In contrast, the metformin concentration in plasma was maintained at low levels within $\sim 600$ min post administration of metformin-PLA-MPs, showed lower $\mathrm{C}_{\max }(1.36 \pm 0.05 \mu \mathrm{g} / \mathrm{ml})$ and higher $\mathrm{T}_{\max }(138 \mathrm{~min})$ than that of metformin oral solution, this may be attributed to the delayed release of metformin from PLA microparticles.

The pharmacokinetic parameters derived from plasma concentration-time data for the different routes are summarized in Table 1. The results indicated that the Mean Residence Time (MRT) was prolonged with PLA microparticles loaded with drug (369.08 $\mathrm{min}$ ) as compared to MRT of pure metformin solution given by IV and oral route respectively (156.52 $\mathrm{min}, 254.59 \mathrm{~min}$ ). This longer MRT enables to maintain the concentration of metformin for prolonged period in rabbit plasma.

Regards the half-life time $\left(t_{1 / 2}\right)$, it was noted that thet $t_{1 / 2}$ of metformin loaded PLA microparticles following oral administration ( $223.30 \pm 21.30 \mathrm{~min}$ ) was higher in comparison to the $t_{1 / 2}$ obtained from pure metformin solution administered by oral $(153.78 \pm 4.19 \mathrm{~min})$ and iv $(139.61 \pm 4.39 \mathrm{~min})$ routes, respectively.

This result indicates that incorporation/encapsulation of metformin in the PLA microparticles achieved high half lifetime.

The $\mathrm{AUC}_{0-\infty}$ after oral administration of metformin PLA microparticles was $617.88 \pm 12.92 \mu \mathrm{g} / \mathrm{ml} / \mathrm{min}$, while $\mathrm{AUC}_{0-\infty}$ of pure metformin solution was $882.85 \pm 23.90 \mu \mathrm{g} / \mathrm{ml} / \mathrm{min}$ and $2440.77 \pm 68.71 \mu \mathrm{g} / \mathrm{ml} / \mathrm{min}$ after oral and IV administration respectively. The oral bioavailability of metformin MPS is calculated to be $25.59 \pm 0.46 \%$. Significantly low $A U_{0-\infty}$ of metformin PLA microparticles indicates low release of metformin from the developed microparticles as compared to oral solution. In the case of the PLA microparticles, the decrease in $A U C_{0-\infty}$ and the low $C_{\text {max }}$ value could be explained by a further decrease in the diffusion of metformin from the PLA microparticles which limit the release of the drug. 
It was also observed that the values of other pharmacokinetics parameters such as $\mathrm{V}_{\mathrm{d}}(2606.33 \pm$ $233.54 \mathrm{~mL} / \mathrm{Kg}), \mathrm{Cl}(8.09 \pm 0.17 \mathrm{~mL} / \mathrm{min} / \mathrm{kg})$ and MAT of metformin encapsulated in PLA microparticles (206.95 $\pm 31.91 \mathrm{~min}$ ) were higher than the pharmacokinetics parameters observed for pure metformin solution after oral and intravenous administration (Table 1).

Table 1

Pharmacokinetic parameters of metformin loaded PLA microparticles and pure drug suspensions from healthy rabbits given a single dose (after single oral administration of $5 \mathrm{mg} / \mathrm{kg}$ body weight to rabbits. Mean \pm S.D. $(n=5)$.

\begin{tabular}{|llll|}
\hline Parameter & IV solution & Oral solution & Oral microparticle \\
\hline $\mathrm{C}_{\max }(\mu \mathrm{g} / \mathrm{ml})$ & - & $3.19 \pm 0,16$ & $1.36 \pm 0.05$ \\
\hline $\mathrm{T}_{\max }(\mathrm{min})$ & - & $110.5 \pm 2.37$ & $138.94 \pm 4.73$ \\
\hline $\mathrm{T}_{1 / 2}(\mathrm{~min})$ & $139.61 \pm 4,39$ & $153.78 \pm 4.19$ & $223.30 \pm 21.30$ \\
\hline $\mathrm{AUC} \mathrm{C}_{-\infty}(\mu \mathrm{g} / \mathrm{ml} / \mathrm{min})$ & $2440.77 \pm 68.71$ & $882.85 \pm 23,90$ & $617.88 \pm 12.92$ \\
\hline $\mathrm{V}_{\mathrm{d}}(\mathrm{mL} / \mathrm{Kg})$ & $412.65 \pm 9.63$ & $1257.59 \pm 58.98$ & $2606.33 \pm 233.54$ \\
\hline $\mathrm{Cl}(\mathrm{mL} / \mathrm{min} / \mathrm{kg})$ & $2.05 \pm 0.06$ & $5.67 \pm 0.15$ & $8.09 \pm 0.17$ \\
\hline $\mathrm{MRT}(\mathrm{min})$ & $156.52 \pm 3,81$ & $254.59 \pm 4.54$ & $369.08 \pm 18.98$ \\
\hline $\mathrm{MAT}(\mathrm{min})$ & - & $98.05 \pm 3.36$ & $206.95 \pm 31.91$ \\
\hline $\mathrm{F}(\%)$ & - & $36.19 \pm 1.27$ & $25.59 \pm 0.46$ \\
\hline
\end{tabular}

\section{Discussion}

The oral route is by far the preferred route of drug administration for many dosages forms. However, many anti-diabetic drugs suffer from low bioavailability following oral administration especially BCS class III like metformin due to its high solubility and low permeability [17]. Therefore, microencapsulation into hydrophobic polymer microparticles systems is an effective strategy to achieve sustained release over time and enhance drug bioavailability in human and veterinary medicine.

In a previous study, we showed the successful preparation of poly (lactic acid) microparticles loaded metformin using the double emulsion ( $\mathrm{w} / \mathrm{o} / \mathrm{w}$ ) solvent evaporation method. In this method, the effect of microencapsulation parameters such as the amount of metformin, $\mathrm{pH}$ of the external aqueous phase, PVA concentration and the stirring rate on the encapsulation efficiency, microparticles size and zeta potential were evaluated and optimized using response surface methodology by Box-Behnken Design [14]. In the present study after microparticles characterization, the potential of poly (lactic acid) microparticles loaded metformin to sustain the in vivo release carried out on rabbits under standard fed conditions. 
Metformin loaded PLA microparticles, under the conditions reported previously, exhibited high encapsulation efficiency $(76 \pm 2.72 \%)$ and spherical shape with no porous surfaces. In the FTIR diagrams, some variations in the absorption bands could be observed befDore and after loading metformin onto the microparticles. These changes could be resulted from the physicochemical interactions between the microparticles and metformin to form metformin-loaded microparticles during the process of microencapsulation [18]. The solid state characteristic is important in controlling drug release. High energy is required in the crystalline states to separate the molecules that led to low aqueous solubility and consequently low physiological bioavailability, while the amorphous state need low energy to separate molecules and drug solubility and bioavailability is superior [10]. SEM images, XRD and DSC results indicate that metformin was dissolved in the microparticles with the presence of intermolecular interactions between the drug and the polymer, and the drug was encapsulated in the microparticles in a non-crystalline state. The crystallinity of metformin and PLA disappeared after microencapsulation because of the fast diffusion of dichloromethane to the outer phase during evaporation step, thus inhibits the formation of crystalline structure [15].

Microencapsulation of metformin in the PLA microparticles caused a change in the pharmacokinetics of the drug in the rabbit model used in this study. $\mathrm{C}_{\max }, \mathrm{T}_{\max }$ and Bioavailability $(\mathrm{F})$ reflect the in vivo absorption rate of drug. In our study, metformin concentration in plasma was maintained at a low level for $24 \mathrm{~h}$ after oral administration of metformin-PLA-MPs, demonstrating slow released metformin which may provide an efficient therapeutic concentration for a prolonged period of time. The peak plasma concentration $\left(C_{\max }=1.36 \pm 0.05 \mu \mathrm{g} / \mathrm{ml}\right.$ ) for metformin-PLA-MPs is 2.34 -fold lower than the peak plasma concentration of oral administration of pure metformin solution. In addition, the time $\left(T_{\max }\right)$ needed to reach the maximum plasma concentration was significantly delayed in the metformin-PLA-MPs group which may be due to the low absorption of metformin in the cells of the gastrointestinal truck. This type of behaviour is expected to PLA microparticles and it has been found for extended release formulations. The mean value of bioavailability (F) obtained in the oral solution and microencapsulated administration groups were $36.19 \%$ and $25.59 \%$, respectively. This value is similar to bioavailability of metformin in diabetic rabbits (36.73\%) [19] and rats (34.1\%) [20], lower than in cats (48\%) [21] and humans (50-60\%) [22]. However, significantly higher than in horses (3.9\%) [23]. The F value of the metformin-PLA-MPs formulation $(25.59 \%)$ is promising showing the characteristic sustained-release of metformin of extended release formulations.

Particle size and size distribution could influence the in vivo dissolution rate as well as the oral bioavailability of a drug. As shown from the SEM image (Fig. 2), PLA microparticles obtained had a large particle size that leads to slower degradation rate of the polymer matrix. As a result, a delayed of the drug diffusion through PLA microparticles occurs, this may explain the lower $\mathrm{C}_{\max }$ and the $\mathrm{AUC}_{0-\infty}$ of the metformin-PLA-MPs compared to the oral solution [24].

The nature of the drugs to encapsulate is an important factor which influences the release of drug from polymer matrix [25]. The experimental finding by IRTF and DSC suggest the presence of interaction between protonated metformin and carboxylic end group of PLA which affect the drug release and led to 
a slow release rate over time. Our results are consistent to those obtained by Proikakis et al. [25]. Besides, the amorphous state of metformin in the microparticles could enhance the release of the drug; however, it is not the case in our study. This could be due to the slow degradation of PLA polymer [26].

Based on these findings, it could be concluded that a promising extended-release PLA microparticles of the highly water soluble drug, metformin, was successfully designed. However, because of the low bioavailability of metformin, future works are needed to improve the bioavailability of the PLA microparticles by evaluating the influence of PLA microparticle's properties on the release of metformin.

\section{Conclusions}

Metformin hydrochloride loaded into PLA microparticles was prepared according to their individual optimised formulations developed in our previous study. The obtained microparticles were spherical with heterogeneous size distribution. The pharmacokinetics parameters resulted in delayed $\mathrm{T}_{\text {max }}$, increased MRT and $t_{1 / 2}$, decreased $\mathrm{C}_{\max }$ of metformin from microparticles that show promise for prolonged/sustained release of metformin after oral administration in different animal species affected by insulin disorders. Low bioavailability is the consequence of slow release of metformin from PLA microparticles. Therefore, further work is needed to investigate the importance of microparticle size distribution on the release from PLA matrix and the release mechanism of these sustained-release microparticles.

\section{Methods}

\section{Materials}

Metformin HCl Mw $165.6 \mathrm{~g} / \mathrm{mol}$. Poly (lactic acid) PLA was purchased from Evonik Industries AG (Germany). Poly (vinyl alcohol) (PVA, 87-89\% hydrolyzed, average $\mathrm{Mw}=30000-70000$ ) was provided by Sigma Aldrich (Barcelona, Spain). High performance liquid chromatography (HPLC) grade acetonitrile, methanol and water were purchased from sigma Aldrich (Barcelona, Spain), Sodium Dodecyl Sulfate (SDS) ultrapure $288.38 \mathrm{~g} / \mathrm{mol}$ and ammonium acetate $77.08 \mathrm{~g} / \mathrm{mol}$ were purchased from (Panreac, Barcelona). All other reagents were of analytical grade.

\section{Animals}

Fifteen healthy New Zealand white female rabbits were obtained from the Faculty of Veterinary of the University of Murcia (Murcia, Spain). The rabbits were housed in cage in laboratory animal rooms of the laboratory of pharmacy, pharmacology and therapeutics of Veterinary faculty. The animals were kept under a photoperiod of $12 \mathrm{~h}$ light/ $12 \mathrm{~h}$ dark. They received standard laboratory chow diet (Nanta, Madrid, Spain) and tap water. All experiments protocols were carried out in accordance with the requirements of applicable national legislation and approved by the Bioethics Committee of the University of Murcia. After 
completion of the study, the animals were donated to be adopted as pets as they all were healthy after physical examination.

\section{Preparation of Metformin HCl-microparticles}

Metformin $\mathrm{HCl}$ loaded PLA microparticles were prepared by the W/O/W solvent evaporation method described previously [14]. Briefly, $1 \mathrm{~mL}$ of aqueous internal phase was emulsified for $5 \mathrm{~min}$ in $10 \mathrm{~mL}$ of methylene chloride (containing $200 \mathrm{mg}$ of PLA) with an ultrasound bath (Branson 5510, BioBlock Scientific, Spain) at $135 \mathrm{~W}$ output. This primary emulsion was gradually added into $40 \mathrm{~mL}$ of a $1.5 \%$ PVA aqueous solution and homogenized for $30 \mathrm{~min}$ at $700 \mathrm{rpm}$ in order to create the W/O/W emulsion. The Solvent evaporation was achieved at room temperature and atmospheric pressure under a $400 \mathrm{rpm}$ agitation (HeidolphHei-Tec D91126, Germany). Microparticles were obtained after centrifugation of the colloidal suspension for $30 \mathrm{~min}$ at $44000 \mathrm{rpm}$ (Centrifuge 5702, Eppendorf Ag, Germany). Drug free microparticles (Placebo MPs) were prepared with the same procedure.

\section{Determination of the encapsulation efficiency (EE)}

The amount of metformin entrapped within polymeric microparticles was determined according to an established High-Performance Liquid Chromatography (HPLC) method by measuring the amount of nonencapsulated metformin in the external aqueous solution, which was recovered after centrifugation of microparticles. The assay was repeated using different samples from independent preparations. The encapsulation efficiency (EE) is defined as the amount of metformin present in the microparticles $\left(m_{1}\right)$ divided by the initial amount added in the inner aqueous phase $\left(\mathrm{m}_{0}\right)$ and it is calculated with the following formulas:

$$
E E(\%)=\mathrm{m}_{1} / \mathrm{m}_{2} \times 100
$$

\section{Microparticles characterisation}

\section{Scanning electron microscopy (SEM)}

The morphology of metformin loaded microparticles was investigated by a scanning electron microscopy (SEM) (Zeiss Evo 50, Germany) at a working distance of $9.5 \mathrm{~mm}$ and accelerating voltage of $8 \mathrm{KV}$. The microparticles were prepared by fixing onto carbon adhesive tape and spotter coating with $10 \mathrm{~nm} \mathrm{Pd} / \mathrm{Au}$ layer under vacuum (EVO/LS 15). The microparticles were examined for shape, size and surface characteristic. 


\section{Fourier transforms infrared (FTIR) spectroscopy}

In order to examine the drug polymer interaction, the FTIR spectra of pure metformin, blank PLA MPs and metformin loaded microparticles were performed from IR Affinity-1 CE spectrophotometer (Shimadzu, Japan). One milligram of samples was finely mixed with $100 \mathrm{mg}$ of purified potassium bromide and pressed in a mechanical die press to form a pellet ( $90 \mathrm{~N}, 5 \mathrm{~min})$. These pellets were scanned, and spectra were recorded from 400 to $4000 \mathrm{~cm}^{-1}$ at a revolution of $4 \mathrm{~cm}^{-1}$.

\section{X-ray powder diffraction (XRPD)}

Pure metformin, blank PLA MPs and metformin loaded microparticles were investigated by wide angle Xray diffraction (XRPD) technique using X-ray diffractometer (X Per-Pro, PANalytical, Netherland). All the samples were scanned at $40 \mathrm{KV}, 30 \mathrm{~mA}$ using Cu-Ka radiation $(\lambda=1.54059 \AA)$ at the range of $(2 \theta)$ from 5 to 80 degree with a scanning speed of $5^{\circ} \mathrm{min}^{-1}$.

\section{Differential scanning calorimetry (DSC)}

The thermal properties of samples were evaluated by differential scanning calorimetry (DSC-131, Setaram, France). DSC thermograms of pure metformin, blank PLA MPs and metformin loaded MPs were obtained using SETSOFT software. The samples $(8-12 \mathrm{mg})$ were weighted and sealed into aluminium pans; an empty sealed pan was used as a reference. DSC curves were obtained at a heating rate of $10{ }^{\circ} \mathrm{C}$ from $30-300^{\circ} \mathrm{C}$.

\section{In vivo Pharmacokinetic study}

Fifteen New Zealand healthy white rabbits $(n=15)$ were selected for the pharmacokinetic study. The rabbits were randomly (Excel random numbers tables generator) divided into 3 groups $(n=5)$ in 3 separated individual and marked cages, and were received a single oral dose administration of metformin microparticles suspension (Group A) pure metformin solution (Group B) by gastric intubation using oral feeding needle respectively, and intravenous (IV) of pure metformin solution by injection from the marginal ear vein (Group C). Investigators were not blinded to sample collection or sample analysis at any stage of the study. There is no hypothesis testing involved in this pharmacokinetics study, and therefore a power analysis is not required for estimating sample size as previously described [27]. The microparticles and pure metformin were suspended/dissolved in saline before each administration at a dose of $5 \mathrm{mg} / \mathrm{kg}$ body weight. At predetermined time points post administration $(10,20,30,45,60,90,120$ minutes and $4,6,8,10,24,34$ and 48 hours), blood samples were extracted $(0.5 \mathrm{~mL})$ from the marginal ear vein of the rabbits by heparinised syringe and immediately centrifuged $(10.000 \mathrm{rpm}, 10 \mathrm{~min})$ to separate plasma that was frozen at $-50^{\circ} \mathrm{C}$ until further analysis. 


\section{Plasma analysis}

To quantify the drug in plasma rabbits a validated method [28] modified by Carceles-Rodriguez et al. [29] was used. For this, samples were thawed. Then, acetonitrile $(200 \mu \mathrm{L})$ was added to plasma $(200 \mu \mathrm{L})$ vortexed for $15 \mathrm{~s}$ then shacked in ultrasonic bath for $5 \mathrm{~min}$ to allow a complete mixing, followed by centrifugation at $12.000 \mathrm{rpm}$ for 10 minutes to extract metformin. Afterwards, $200 \mu \mathrm{l}$ of the supernatant was mixed to HPLC mobile phase (ratio of 1:3) transferred into HPLC vials and analyzed. The pharmacokinetic parameters were calculated using a non-compartmental model.

\section{HPLC method}

HPLC analysis was performed using a JASCO series HPLC and a JASCO 1575 UV/VIS detector set at a wavelength of $236 \mathrm{~nm}$. The chromatographic separation was achieved by a Kromasil C-18 reverse-phase column ( $250 \times 4.6 \mathrm{~mm}$ of $5 \mu \mathrm{m}$, (AnálisisVínicos S.L., C. Real, Spain) at $25^{\circ} \mathrm{C}$. The mobile phase employed was acetonitrile: ammonium acetate $(25 \mathrm{mM})$ - dodecyl sulphate sodium $(9 \mathrm{mM})(\mathrm{pH} 7.02)$ in a volume ratio of 45:55. The flow rate and injection volume of metformin were $1 \mathrm{~mL} / \mathrm{min}$ and $100 \mu \mathrm{L}$, respectively. A standard calibration curve was performed with metformin in plasma. The established linearity range was $100-10000 \mu \mathrm{g} / \mathrm{L}(r>0.99)$. The duration of the analysis is $10 \mathrm{~min}$ and the retention time of metformin is approximately $5 \mathrm{~min}$.

\section{Pharmacokinetics analysis}

The plasma metformin time-concentration data were analyzed by non-compartment methods. The Area Under the Concentration-time Curve $\left(\mathrm{AUC}_{0-\infty}\right)$ was calculated using the linear trapezoidal rule with extrapolation to infinity. Mean Residence Time was calculated as MRT = AUMC/AUC. The systemic clearance as $\mathrm{Cl}=$ Dose/AUC. The apparent volume of distribution (area method) and apparent volume of distribution at steady state were calculated as $V_{z}=$ Dose $/\left(A U C \cdot I_{z}\right)$ and $V_{s s}=($ Dose $\cdot A U M C) / A^{\prime} C^{2}$, respectively.

\section{Statistical analysis}

Descriptive statistical parameters as mean, standard deviation (SD) and coefficient of variation (CV) were calculated. Harmonic means were calculated for the half-lives of elimination and absorption. KruskalWallis test was used to check for normal distribution of parameters and concentrations ranges between animals. The Wilcoxon Rank Sum and Student's $t$ tests were used to test parameters for significant differences between the different routes of administration. The statistical software used was SPSS Version 19.0 (SPSS Statistic Programme, Chicago, USA). Values of $P<0.05$ were considered significant.

\section{Abbreviations}


PLA: poly lactic acid; $T_{\text {max }}$ : time to reach maximum concentrations; $C_{\text {max }}$ : maximum concentrations; MRT: Mean Residence Time; BCS: Biopharmaceutics Classification System class III drug; CDDSs: polymeric controlled drug delivery systems; SEM: scanning electron microscopy; FTIR: Fourier transforms infrared spectroscopy; XRPD: X-ray powder diffraction; DSC: Differential scanning calorimetry; HPLC: Hig Performance Liquid Chromatography; EE: Determination of the encapsulation efficiency; $\mathrm{AUC}_{0-\infty}$ : Area Under the Concentration-time Curve; SD: Standard deviation; CV: coefficient of variation.

\section{Declarations}

\section{Ethics approval and consent to participate}

All experiments protocols were carried out in accordance with the requirements of applicable national legislation and approved by the Bioethics Committee of the University of Murcia.

\section{Consent for publication}

Not applicable.

\section{Availability of data and material}

Research data are available as supplementary files.

\section{Competing interest}

The authors declare that they have no conflict of interest.

\section{Funding}

This research was funded by the Government of the Region de Murcia (Spain) by the Fundación Séneca (project 20950/PI/18). Fundación Séneca had no role in the design of the study, collection, analysis, interpretation of data and in writing the manuscript.

\section{Authors' contributions}

SB: Investigation, writing. AAG: Investigation. CMCR: Investigation, writing, statistical analysis, Pharmacokinectis analysis. FR: Conceptualization, writing. EFV: Funding acquisition, investigation, project administration, writing. All authors have read and approved the manuscript. 


\section{Acknowledgements}

Not applicable.

\section{References}

1. International Diabetes Federation. 8th edition. 2017.

2. Nelson RW. Oral medications for treating diabetes mellitus in dogs and cats. J Small Anim Pract. 2000;41(11):486-90.

3. Conaway HH, Faas FH, Smith SD, Sanders LL. Spontaneous diabetes mellitus in the New Zealand white rabbit: physiologic characteristics. Metabolism. 1981;30(1):50-6.

4. Cárceles-Rodríguez CM, Fernández-Varón E, Martín-Gimenez T, Aguirre $C$, Arion A, Rodríguez MJ, Ayala I. Pharmacokinetics of Metformin in Combination With Sitagliptin in Adult Horses After Enteral Administration. J Equine Vet Sci. 2019;72:84-8.

5. Hustace JL, Firshman AM, Mata JE. Pharmacokinetics and bioavailability of metformin in horses. Am J Vet Res. 2009;70:665-8.

6. Creight $L J$ MC, Bailey $C J$, Pearson ER. Metformin and the gastrointestinal tract. Diabetologia 2016;59:426-435.

7. Martínez-Gómez F, Guerrero J, Matsuhiro B, Pavez J. In vitro release of metformin hydrochloride from sodiumalginate/polyvinyl alcohol hydrogels. Carbohydr Polym. 2017;155:182-91.

8. Chinnaiyan SK, Deivasigamani K, Gadela VR. Combined synergetic potential of metformin loaded pectin-chitosan biohybrids nanoparticle for NIDDM. Int J Biol Macromol. 2019;125:278-89.

9. Shariatinia Z, Zahra Z. Controlled release of metformin from chitosan-based nanocomposite films containing mesoporous MCM-41 nanoparticles as novel drug delivery systems. J Colloid Interface Sci. 2017;501:60-76.

10. Altmeyer C, Ksiaskiewcz Karam T, Maissar Khalil N, Mainardes RM. Tamoxifen-loaded poly (Llactide) nanoparticles: Development, characterization and in vitro evaluation of cytotoxicity. Mater Sci Eng C. 2016;60:135-42.

11. Thauvin C, Schwarz B, Delie F, Allémann E. Functionalized PLA polymers to control loading and/or release properties of drug-loaded nanoparticles. Int J Pharm. 2018;548:771-7.

12. Lee BK, Yun Y, Park K. PLA micro- and nano-particles. Advanced Drug Deliv Rev. 2016;107:176-91.

13. Pastorino L, Dellacasa E, Petrini P, Monticelli O. Stereo complex poly (lactic acid) nanocoated chitosan microparticles for the sustained release of hydrophilic drugs. Mat Sci Eng C. 2017;76:112935.

14. Bouriche S, Cózar-Bernal MJ, Rezgui F, Rabasco Álvarez AM, González-Rodríguez ML. Optimization of preparation method by W/O/W emulsion for entrapping metformin hydrochloride into poly (lactic acid) microparticles using Box Behnken design. J Drug Deliv Sci Tech. 2019;51:419-29. 
15. Hyvönen S, Peltonen L, Karjalainen M, Hirvonen J. Effect of nanoprecipitation on the physicochemical properties of low molecular weight poly (I-lactic acid) nanoparticles loaded with salbutamol sulphate and bec lomethasonedipropionate. Int J Pharm. 2005;295:269-81.

16. Liu H, He J. Simultaneous release of hydrophilic and hydrophobic drugs from modified chitosan nanoparticles. Mater Lett. 2015;161:415-8.

17. Uppal S, Italiya KS, Chitkara D, Mittal A. Nanoparticulate-based drug delivery systems for small molecule anti-diabetic drugs: An emerging paradigm for effective therapy. Acta Biomater. 2018;81:20-42.

18. Barbara S, Infrared Spectroscopy. In Encyclopedia of Chemical Technology; Othmer K, E.d.; John Wiley \& Sons: New York; 2015; pp1-17.

19. Díez R, García JJ, Diez MJ, Sierra M, Sahagun AM, Fernández N. Influence of Plantago ovata husk (dietary fiber) on the bioavailability and other pharmacokinetic parameters of metformin in diabetic rabbits. BMC Complement Altern Med. 2017;17:298-1-9.

20. Choi Y, Chung S, Lee M. Pharmacokinetic interaction between DA-8159, a new erectogenic, and metformin in rats: competitive inhibition of metabolism via hepatic CYP3A1/2. British J Pharmacol. 2009;153(7):1568-78.

21. Michels GM, Boudinot FD, Ferguson DC, Hoenig M. Pharmacokinetics of the antihyperglycemic agent metformin in cats. Am J vet Res. 1999;60:738-42.

22. Tucker GT, Casey C, Phillips PJ, Connor H, Ward JD, Woods HF. Metformin kinetics in healthy subjects and in patients with diabetes mellitus. Br J Clin Pharmacol. 1981;12:235-46.

23. Hustace JL, Firshman AM, Mata JE. Pharmacokinetics and bioavailability of metformin in horses. Am J vet Res. 2009;70:665-72.

24. Guo C, Chen Y, Zhu J, Wang J, Xu Y, Luan H, Zhu Z, Hu M, Wang H. Preparation, optimization of intravenous ZL-004 nanosuspensions by the precipitation method, effect of particle size on in vivo pharmacokinetics and tissue distribution. J Drug Deliv Sci Tec. 2019;50:313-20.

25. Proikakis CS, Tarantili PA, Andreopoulos AG. The role of polymer/drug interactions on the sustained release from poly (DL-lactic acid) tablets. Eur Polym J. 2006;42:3269-76.

26. Zhang P, Chen L, Gu W, Xu Z, Gao Y, Li Y. In vitro and in vivo evaluation of donepezil-sustained release microparticles for the treatment of Alzheimer's disease. Biomaterials. 2007;28:1882-8.

27. Riviere JE. Comparative Pharmacokinetics: Principles, Techniques, and Principles. Second ed. Hoboken: Wiley-Blackwell; 2011. pp. 1-443.

28. Ross MS. Determination of metformin in biological fluids by derivatization followed by highperformance liquid chromatography. J Chromatogr A. 1977;133:408-11.

29. Carceles-Rodriguez CM, Fernández-Varón E, Martín-Gimenez T, Aguirre C, Arion A, Rodríguez MJ, Ayala I. Pharmacokinetics of metformin in combination with sitagliptin in adult horses after enteral administration. J Equine Vet Sci. 2019;72:84-8. 
Figures

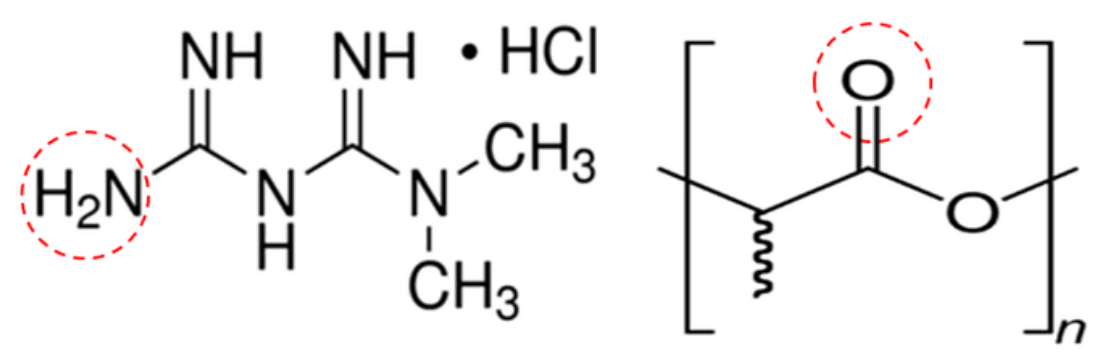

Figure 1

Chemical structure of metformin (a), PLA (b) and their potent groups for hydrogen bonding formation. 


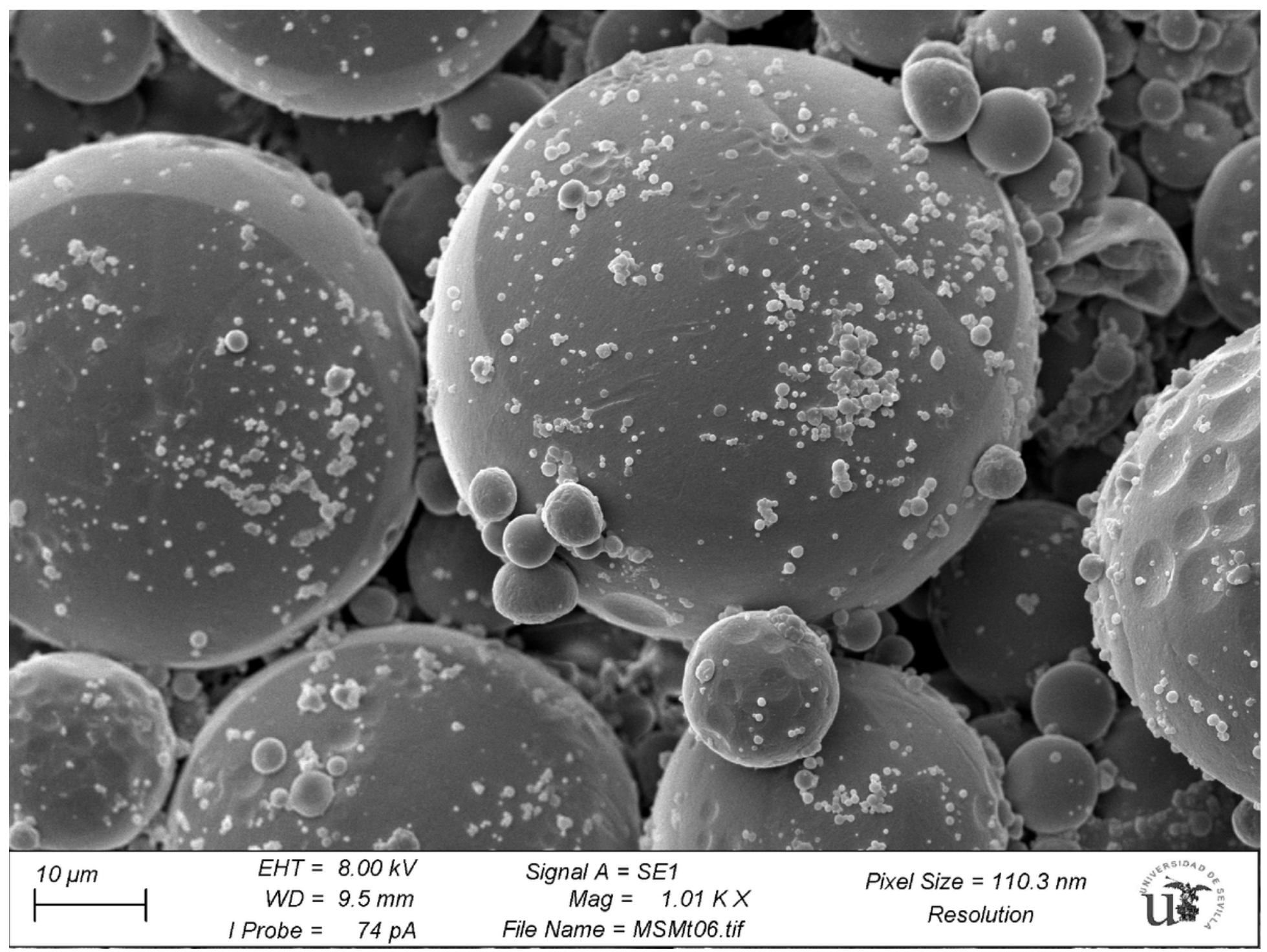

Figure 2

SEM picture of surfaces of the investigated metformin loaded PLA microparticles. Microparticles had almost spherical shape with smooth surfaces with particles adhered to the surface. The size of microparticles is variable, it is ranged from $\sim 1$ to $55 \mu \mathrm{m}$. 


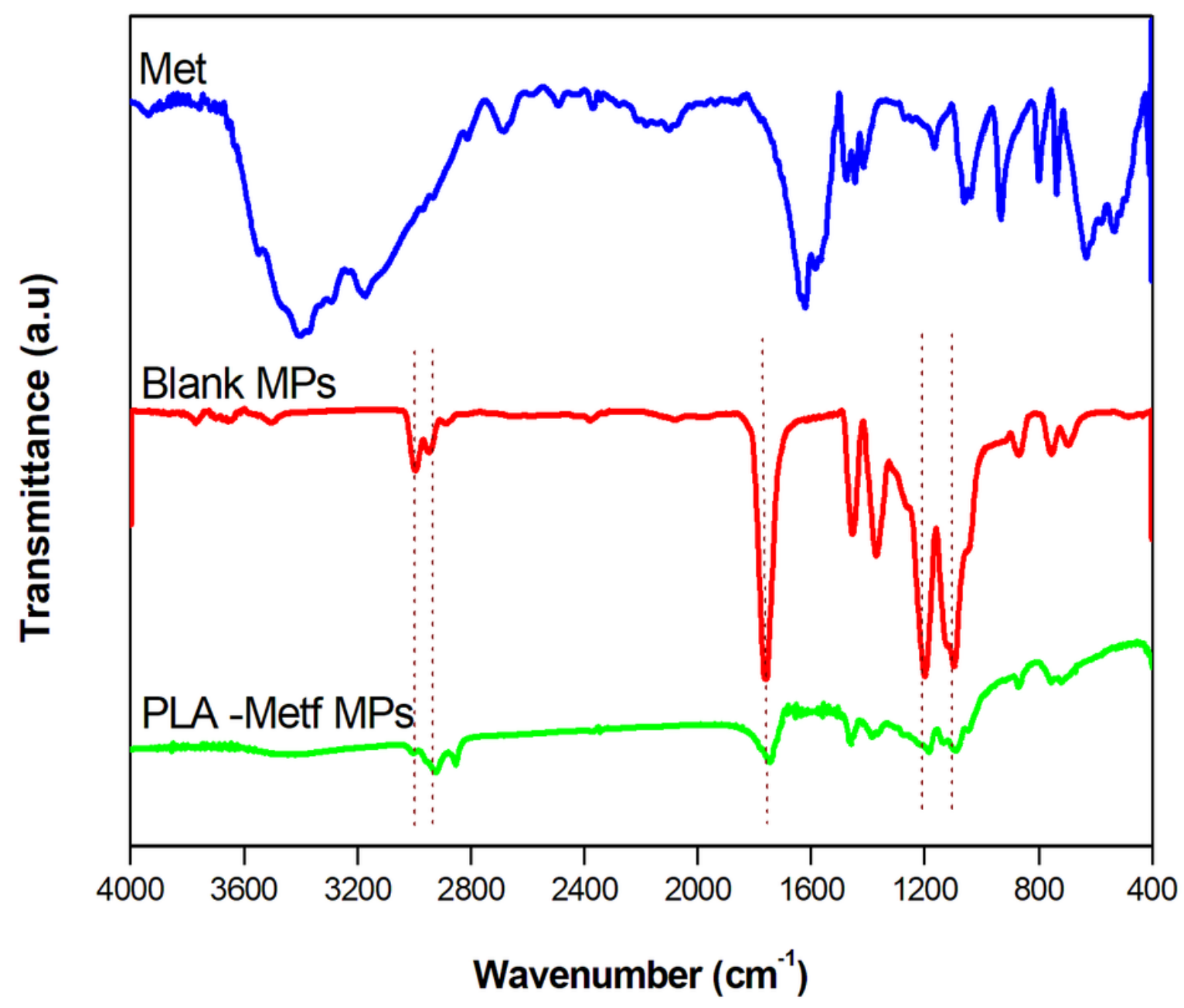

Figure 3

FTIR spectra for pure metformin, blank MPs and metformin loaded microparticles. The FTIR spectrum of pure metformin (blue line) shows characteristic peaks at 3390, 1633 and $1045 \mathrm{~cm}-1$ corresponds to the stretching of primary $\mathrm{N}-\mathrm{H}$, stretching of $\mathrm{C}=\mathrm{N}$, and stretching of $\mathrm{C}-\mathrm{N}$ respectively. For the blank MPs (red line) four characteristic peaks were observed at 1764, 2993-2943 and 1087 which are attributed to $\mathrm{C}=0$ stretching of the ester group, asymmetric and symmetric vibration of the $\mathrm{CH} 3$ group - $\mathrm{CH} 3$ (asym), $\mathrm{CH} 3$ (sym) and C-O ester bond.The microparticles spectra (green line) showsthe intensity of the peak of $\mathrm{C}=0$ ester groups of PLA at $1764 \mathrm{~cm}-1$ after metformin loading was reduced. 


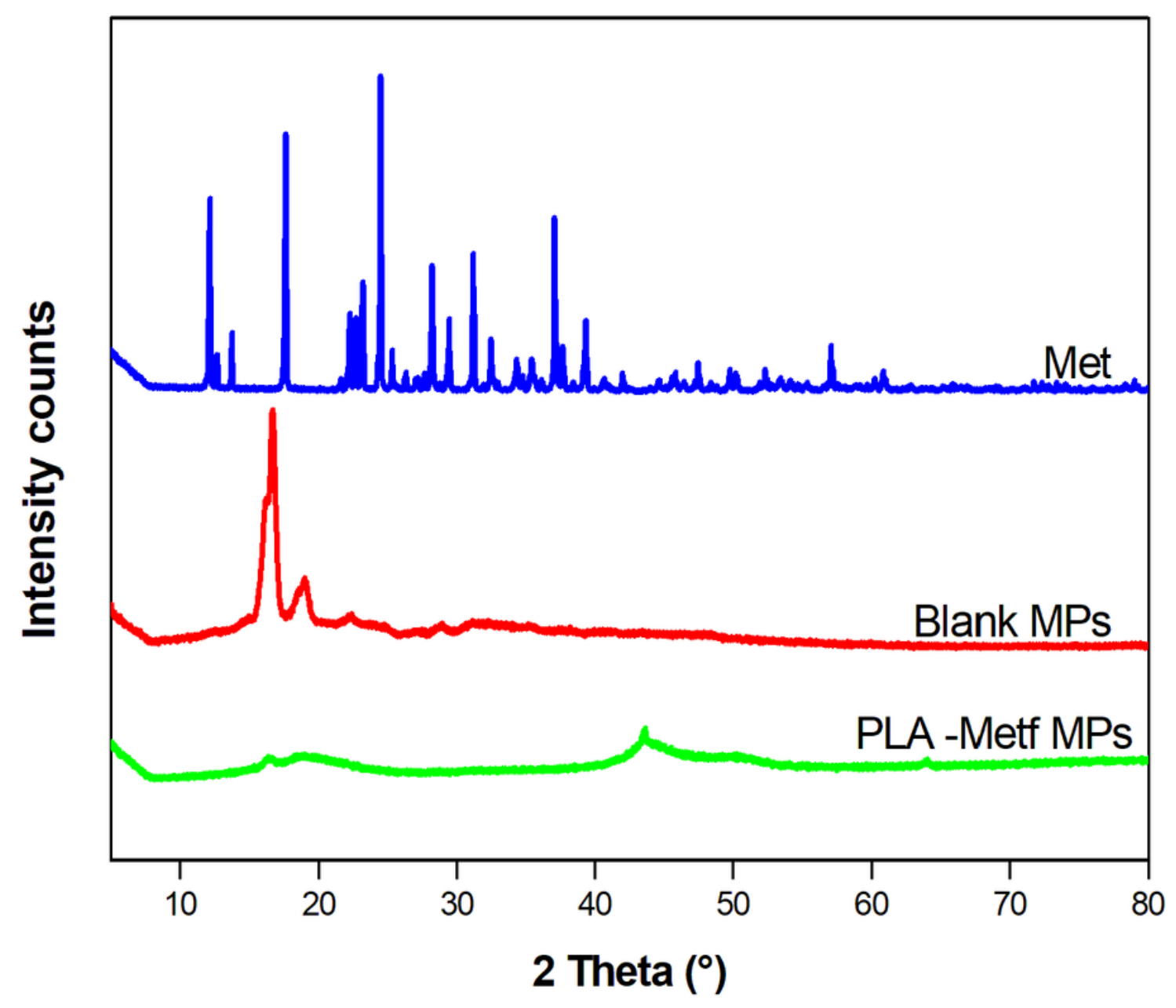

Figure 4

XRD spectra for pure metformin, blank MPs and metformin loaded microparticles. The PXRD

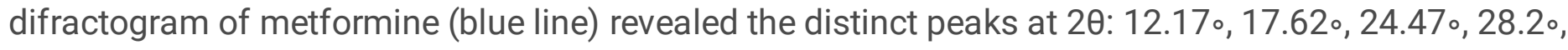
$31.17 \circ, 37.07 \circ$; these sharps peaks confirmed the highly crystalline nature of metformin. Blank MPs (red line) showed the peaks at 20: 16.6。, 18.92。 indicating the semi crystalline nature of the PLA. The PXRD difractogram of MPs (green line) shows no peaks. 


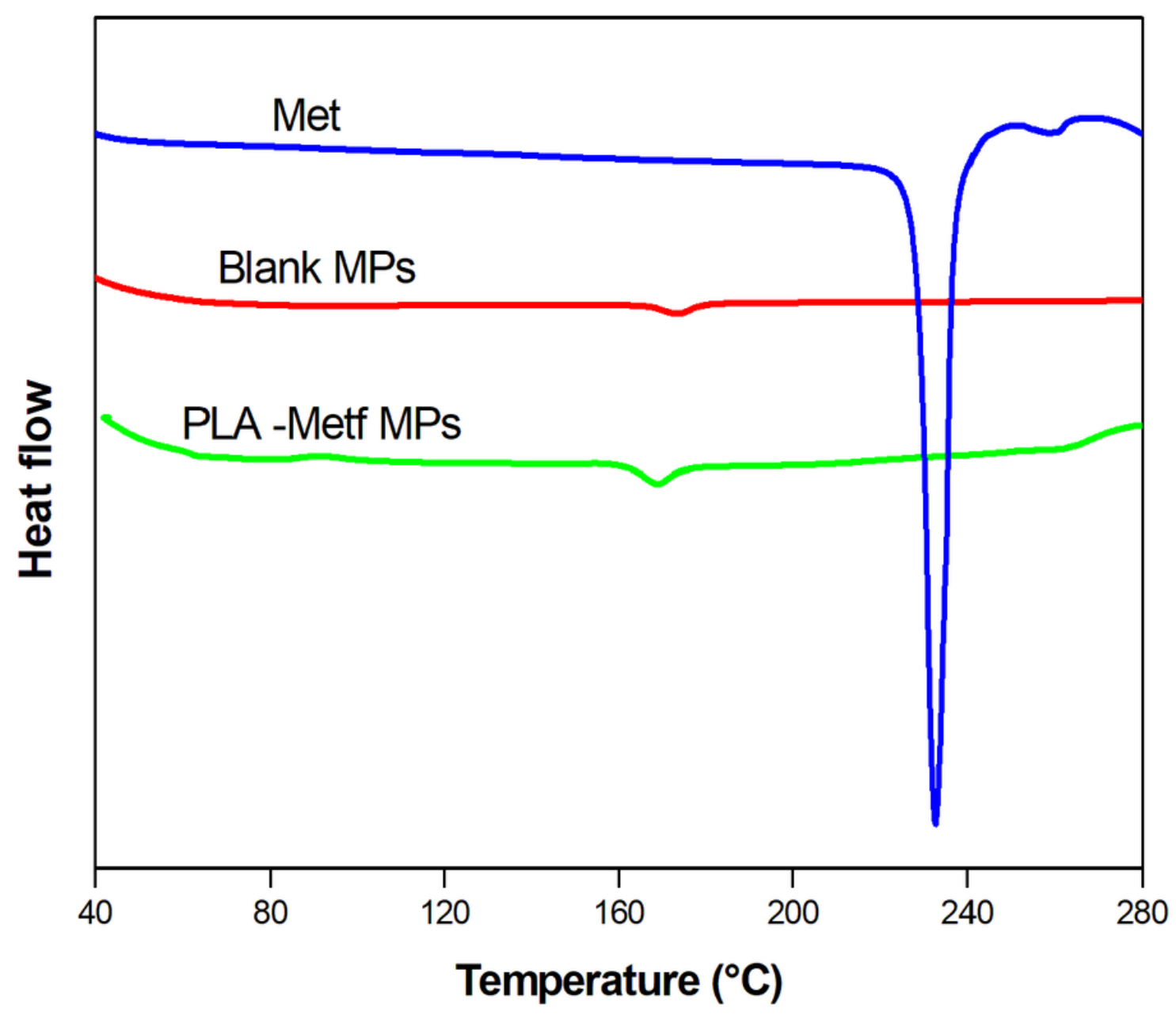

Figure 5

DSC spectra for pure metformin, blank MPs and metformin loaded microparticles. The DSC curves of samples were recorded during the first heating process. The metformin DSC curve (blue line) exhibited a very sharp endothermic peak at $\sim 232{ }^{\circ} \mathrm{C}$ that indicates its crystalline nature. DSC curve of the blank PLA (unloaded) microparticles (red line) showed a broad peak at $\sim 178{ }^{\circ} \mathrm{C}$, while this peak is shifted to $168^{\circ} \mathrm{C}$ in metformin loaded PLA microparticles (green line). No peak of metformin in the loaded microparticles was recorded. 


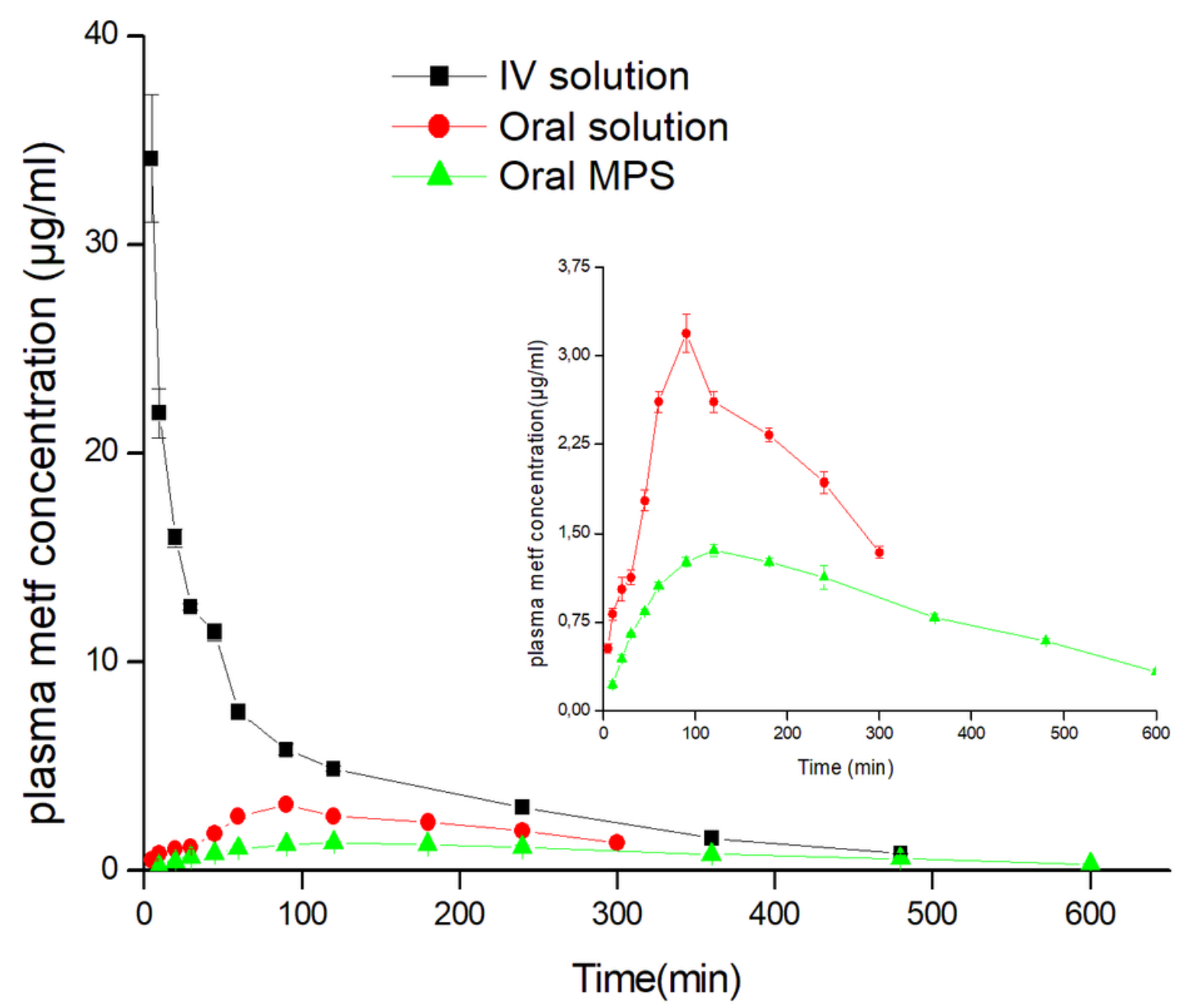

Figure 6

Mean plasma concentration-times profiles of metformin following a single dose administration of its intravenous,oral solution and oral microparticles in rabbits $(n=5)$. The dose of the drug administered was $5 \mathrm{mg} / \mathrm{kg}( \pm S D)$.

\section{Supplementary Files}

This is a list of supplementary files associated with this preprint. Click to download.

- ArrriveAuthorChecklistFullmanuscript.pdf

- Suplementarydata.docx 